\title{
A THEOREM ON THE REPRESENTATION OF POTENTIAL FLOWS HAVING UNBOUNDED FREE SURFACES*
}

By CHARLES F. WEBER (U. S. Naval Ordnance Laboratory, White Oak, Silver Spring, Maryland)

In hydrodynamics problems involving a constant pressure free surface one often seeks an approximate solution by linearizing the boundary condition. In three dimensions, when the free surface displacement from a flat surface is small and the velocity is small in some sense, we replace the surface with a plane and apply the condition $\phi=$ constant on it. If the free surface is infinite and asymptotically planar, and the disturbance local (i.e., there exists $R(t)$ such that in the region $r>R$, where $r$ is a radial spherical coordinate, the only boundary points of the domain in question are those of the free surface), the harmonic function may be continued across the $\phi=0$ free surface as an odd function. The singularities of $\phi$ are then contained wholly within the sphere $r \leq R(t)$.

We pose the question as to whether the singularities of the continuation of the solution of the nonlinear problem also lie in the finite part of space. An essentially negative answer is given by the

Theorem. Consider an incompressible (nonsteady) potential flow with a free boundary extending to infinity. Suppose the flow is axially symmetric, the free surface initially planar and at rest, and the potential well enough behaved so that the Bernoulli equation applies on the surface. Suppose there is a disturbance which is, at any fixed time, local (as defined above), with no external forces. Then, under these circumstances, the potential $\phi$ cannot be represented by a collection of singularities in the finite part of space, for $t$ small enough.

Proof. Suppose $\phi$ had such a representation. We will show that this assumption leads to a contradiction. Under this supposition, $\phi$ possesses an expansion in spherical harmonics which converges uniformly and absolutely in $r>R$, where all the singularities are in the region $r<R$. Write this expansion as

$$
\phi=\sum_{n=1}^{\infty} \frac{A_{n}(t) P_{n-1}}{r^{n}}
$$

where $P_{n-1}(\sin \theta)$ is the Legendre polynomial with the $z=0$ plane corresponding to $\theta=0$, the $z$-axis being the axis of symmetry.

Lemma. The lowest order term cannot correspond to $n$ odd.

Proof. Assume the lowest order term corresponds to $n=p$ odd. Bernoulli's equation on the free surface is

$$
\phi_{t}+\frac{1}{2}(\nabla \phi)^{2}=0,
$$

with the velocity taken as $\nabla \phi$. The domain in which $\phi$ is regular harmonic is taken to lie below the free surface, which is initially at $z=0$.

Now

$$
\phi_{t} \sim \frac{A_{p}^{\prime} P_{p-1}}{r^{p}}, \quad \phi_{r} \sim \frac{-p A_{p} P_{p-1}}{r^{p+1}}
$$

*Received October 23, 1967; revised version received March 26, 1968. 
and

$$
\frac{1}{r} \phi_{\theta} \sim \frac{A_{p} P_{p-1 \theta}}{r^{p+1}},
$$

where the prime indicates time derivative. Since $P_{p-1} \sim$ constant $+O\left(\theta^{2}\right)$, we have, substituting into the Bernoulli equation,

$$
\frac{A_{p}^{\prime} P_{p-1}}{r^{p}}+\cdots+\frac{1}{2}\left(\frac{p^{2} A_{p}^{2} P_{p-1}^{2}}{r^{2 p+2}}+\cdots\right)=0,
$$

where $p$ is an odd integer. Thus $A_{p}^{\prime}=0$. But since the flow begins from rest, this implies that $A_{p}=0$, contradicting the assumption of the lemma.

We will now show that the dominant term cannot correspond to $n$ even, either, thus reaching our contradiction.

Assume the dominant term is

$$
\frac{A_{n} P_{p-1}}{r^{\prime}}
$$

with $p$ even. Write

$$
P_{p-1}=s \sin \theta+O\left(\theta^{3}\right),
$$

where $s$ is a constant. Then

$$
\frac{1}{r} \phi_{\theta}=\frac{A_{p^{s}}+O\left(\theta^{2}\right)}{r^{p+1}}+O\left(\frac{1}{r^{p+2}}\right)
$$

and

$$
\phi_{r}=\frac{-p A_{p} P_{p-1}}{r^{p+1}}+O\left(\frac{1}{r^{p+2}}\right),
$$

where the $O\left(\theta^{2}\right)$ and $O\left(1 /\left(r^{p+2}\right)\right)$ refer to limits as $\theta \rightarrow 0$ and $r \rightarrow \infty$, respectively. Considering that $\theta=o(1 / r)$ on the free surface, the dominant term for the velocity on the free surface in its asymptotic expansion is

$$
A_{p} s /\left(r^{p+1}\right)+o\left(1 /\left(r^{p+1}\right)\right),
$$

where $o$ now refers to the limit as $r \rightarrow \infty$ along the surface. It follows that

$$
z \sim K s /\left(r^{p+1}\right) \quad \text { or } \quad \theta \sim K s /\left(r^{p+2}\right)
$$

on the free surface, where

$$
K=\int_{0}^{\iota} A_{p}(\tau) d \tau,
$$

so that for small enough times, since $A_{p}(0)=0, A_{\nu}^{\prime} K>0$. Therefore, substituting these relationships into (1), we have

$$
\phi_{t} \sim A_{\nu}^{\prime} s^{2} K /\left(r^{2 p+2}\right)+o\left(1 /\left(r^{2 p+2}\right)\right) .
$$

Note that there is no other $1 / r^{2 p+2}$ term in the $\phi_{t}$ expansion, since the even Legendre polynomials appear together with odd powers of $r$ in the spherical harmonic expansion for $\phi$. It is at this point, incidentally, that an attempted proof in two dimensions breaks down, although the lemma still holds [1]. 
Using these asymptotic relationships in the Bernoulli equation gives

$$
r^{2 p+2}\left[\frac{A_{p}^{\prime} K s^{2}}{r^{2 p+2}}+\frac{1}{2} \frac{A_{p}^{2} s^{2}}{r^{2 p+2}}+o\left(\frac{1}{r^{2 p+2}}\right)\right] \rightarrow 0,
$$

so that

$$
A_{p}^{\prime} K+\frac{1}{2} A_{p}^{2}=0 .
$$

This implies $A_{p}^{\prime} K<0$, which contradicts (2), or else $A_{p}=0$, which contradicts the assumption of dominance.

The conditions of this theorem are not very restrictive. Further, Professor A. G. Mackie has shown first how the result can be extended to allow for a gravity term, and second that the assumption of a dominant dipole term leads to a contradiction even in the case of no axial symmetry [2]. It is reasonable to expect, moreover, that if, under the ideal conditions of this theorem, an expansion in spherical harmonics is impossible, it is also impossible under less ideal conditions (save possibly for some very special cases).

It therefore appears that the introduction of the nonlinear terms of the Bernoulli equation together with the true surface shape, affects the nature of the continuation of the potential function into the upper half-space in a nontrivial way. Whereas a multipole expansion may be used to represent $\phi$ for the linearized problem in a half-space and also for an exact nonlinear problem in the full space, e.g., the problem of a bubble expanding from rest in a fluid filling all of space, it is not permitted for the exact problem in the half-space. The lesson to be learned, it seems, is that careful consideration is called for before it is attempted to represent harmonic functions, for the types of problems discussed here, by a bounded system of singularities.

The author is indebted to Professor A. G. Mackie for several useful discussions of these matters and to the Naval Ordnance Laboratory for funding this work as part of an effort in water entry problems.

\section{REFERENCES}

[1] A. G. Mackie, The water entry problem, University of Maryland Technical Note, BN-496

[2] A. G. Mackie, private communication 